\title{
RLS-Adaptive Parallel Interference Cancellation Assisted Decision-Directed Channel Estimation for OFDM
}

\author{
M. Münster and L. Hanzo ${ }^{1}$ \\ Dept. of ECS, Univ. of Southampton, SO17 1BJ, UK. \\ Tel: +44-1703-593 125, Fax: +44-1703-594 508 \\ Email:1h@ecs.soton.ac.uk; http://www-mobile.ecs.soton.ac.uk
}

\begin{abstract}
OFDM systems employing multiple transmit antennas have recently drawn wide interest in the context of both space-time coded- and multi-user space-division multiple access (SDMA) arrangements. A prerequisite for using coherent detection at the receiver is the availability of reliable channel transfer factor estimates. Robust parallel interference cancellation (PIC) assisted decision-directed channel estimation (DDCE) has been shown in the literature to be also applicable to scenarios, where the number of users is in excess of the number of OFDM subcarriers - normalized to the number of Channel Impulse Response (CIR) related taps to be estimated - which imposed a limitation in the context of least-squares assisted DDCE techniques invoked in conjunction with multiple transmit antennas. In this paper we will demonstrate that the Recursive Least-Squares (RLS) algorithm is applicable to optimizing the predictors' coefficients on a CIR-related tap-by-tap basis. Compared to 'robust', non-adaptive approaches the proposed solution has the advantage of a potentially lower estimation MSE and a higher resilience to erroneous subcarrier symbol decisions.
\end{abstract}

\section{OVERVIEW}

The structure of this paper is as follows. In Section II the SDMA signal model as observed on a subcarrier basis in the context of supporting multiple OFDM users with the aid of multiple transmit antennas is outlined. This is followed in Section III by a review of the PIC-assisted DDCE's design. In Section IV we will then describe the RLS-based Channel Impulse Response (CIR) related tap prediction filtering to be used in the context of Decision Directed Channel Estimation (DDCE) contrived for single-user OFDM systems and for Parallel Interference Cancellation (PIC) assisted DDCE designed for OFDM systems employing multiple transmit antennas. The assessment of the estimator's mean-square error (MSE) and the system's bit error-ratio (BER) performance is carried out in Section V. Our conclusions will be offered in Section V-C.

\section{The SDMA Signal Model on A Subcarrier Basis}

In Figure 1 we have portrayed an SDMA uplink transmission scenario, where each of the $P$ simultaneous users is equipped with a single transmission antenna. By contrast, the receiver uses a $P$-element antenna front-end. The set of complex signals, $x_{p}[n, k], p=1, \ldots, P$ received by the $P$-element antenna array in the $k$-th subcarrier of the $n$-th OFDM symbol is constituted by the superposition of the independently faded frequency-domain signals associated with the $L$ users sharing the same space-frequency resource. The received signal was corrupted by the Gaussian noise encountered at the array elements. Regarding the statistical properties of the different signal components

The financial support of the European Union under the auspices of the PanEuropean TRUST project and that of the EPSRC, Swindon UK is gratefully acknowledged

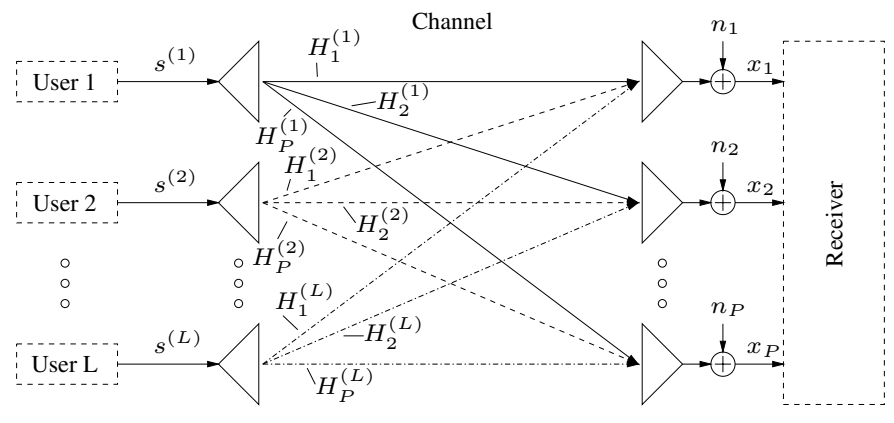

Fig. 1. Schematic of an SDMA uplink scenario as observed on an OFDM subcarrier basis, where each of the $L$ users is equipped with a single transmit antenna and the basestation's receiver is assisted by a $P$-element antenna frontend. For comparison, in an STC scenario the $L$ transmit antennas are used for providing $L$-th order transmit diversity for a single user.

depicted in Figure 1, we assume that the complex data signal $s^{(l)}$ transmitted by the $l$-th user has zero-mean and a variance of $\sigma_{l}^{2}$. The AWGN noise process $n_{p}$ at any antenna array element $p$ exhibits also zeromean and a variance of $\sigma_{n}^{2}$, which is identical for all array elements. The frequency-domain channel transfer factors $H_{p}^{(l)}$ of the different array elements $p=1, \ldots, P$ or users $l=1, \ldots, L$ are independent, stationary, complex Gaussian distributed random variables with zeromean and unit variance.

\section{Parallel Interference Cancellation Assisted DECision-Directed CHANNEl Estimation}

\section{A. A Priori and A Posteriori Channel Estimates}

The complex output signal $x_{p}[n, k]$ of the $p$-th receiver antenna element in the $k$-th subcarrier of the $n$-th OFDM symbol is given by:

$$
x_{p}[n, k]=\sum_{i=1}^{L} H_{p}^{(i)}[n, k] s^{(i)}[n, k]+n_{p}[n, k],
$$

where the different variables have been defined in Section II. Upon invoking vectorial notation, Equation 1 can be rewritten as:

$$
\mathbf{x}_{p}[n]=\sum_{i=1}^{L} \mathbf{S}^{(i)}[n] \mathbf{H}_{p}^{(i)}[n]+\mathbf{n}_{p}[n],
$$

where $\mathbf{x}_{p}[n] \in \mathbb{C}^{K \times 1}, \mathbf{H}_{p}^{(i)}[n] \in \mathbb{C}^{K \times 1}$ and $\mathbf{n}_{p}[n] \in \mathbb{C}^{K \times 1}$ are column vectors hosting the subcarrier-related variables $x_{p}[n, k]$, $H_{p}^{(i)}[n, k]$ and $n_{p}[n, k]$, respectively, and $\mathbf{S}^{(i)}[n] \in \mathbb{C}^{K \times K}$ is a diagonal matrix having elements given by $s^{(i)}[n, k]$, where $k=0, \ldots, K-$ 1. An a posteriori (apt) estimate $\tilde{\mathbf{H}}_{a p t}^{(j)}[n] \in \mathbb{C}^{K \times 1}$ of the vector 


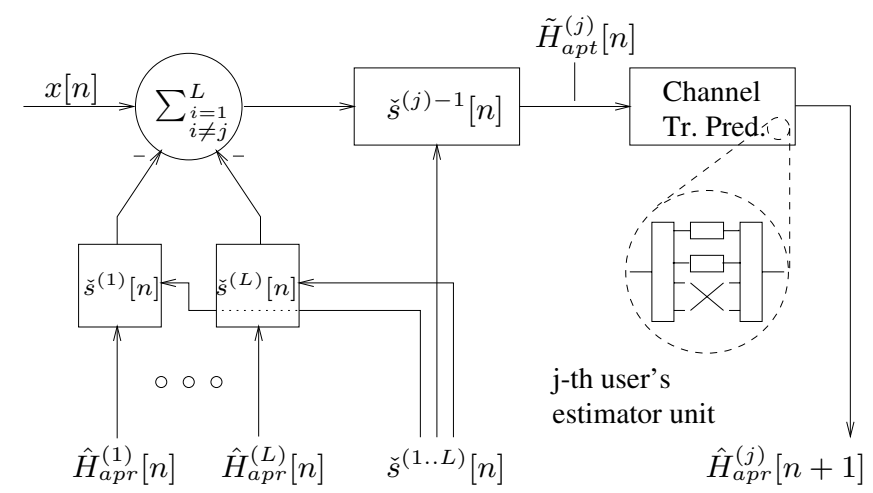

Fig. 2. Illustration of the PIC-assisted channel transfer function estimationor prediction block, associated with the $j$-th user and any of the $P$ receiver antenna elements. The PIC process is described by Equation 3.

$\mathbf{H}^{(j)}[n]$ of 'true' channel transfer factors between the $j$-th user's single transmit antenna and the $p$-th receiver antenna can be obtained by subtracting all the $(L-1)$ vectors of interfering users' estimated signal contributions from the vector $\mathbf{x}_{p}[n]$ of composite received signals of the $L$ users, followed by normalization with the $j$-th user's diagonal matrix of detected complex symbols $\check{\mathbf{S}}^{(j)}[n]$, yielding:

$$
\tilde{\mathbf{H}}_{a p t}^{(j)}[n]=\check{\mathbf{S}}^{(j)-1}[n]\left(\mathbf{x}[n]-\sum_{\substack{i=1 \\ i \neq j}}^{L} \check{\mathbf{S}}^{(i)}[n] \hat{\mathbf{H}}_{a p r}^{(i)}[n]\right),
$$

where for simplicity's sake we have omitted the receiver antenna's in$\operatorname{dex} p$. The PIC process based on Equation 3, has been further illustrated in Figure 2. In Equation 3, $\hat{\mathbf{H}}_{a p r}^{(i)}[n] \in \mathbb{C}^{K \times 1}$ denotes the $i$-th user's vector of complex a priori (apr) channel transfer factor estimates predicted during the $(n-1)$-th OFDM symbol period for the $n$-th OFDM symbol, as a function of the vectors of a posteriori channel transfer factor estimates $\tilde{\mathbf{H}}_{a p t}^{(i)}[n-n]$ associated with the previous $N_{\text {tap }}^{[t]}$ number of OFDM symbols, which is formulated as:

$$
\hat{\mathbf{H}}_{a p r}^{(i)}[n]=f\left(\tilde{\mathbf{H}}_{a p t}^{(i)}[n-1], \ldots, \tilde{\mathbf{H}}_{a p t}^{(i)}\left[n-N_{t a p}^{[t]}\right]\right) .
$$

We will further elaborate on the specific structure of the predictor in the next section.

\section{B. A Priori Channel Prediction Filtering}

The channel transfer function prediction along the time-direction follows the philosophy of the 2D-MMSE channel transfer function estimation approach proposed by Li et al. [1], which in turn is based on the rank-reduction assisted 1D-MMSE channel estimation scheme proposed by Edfors et al. [2].

- In a first step, in order to obtain the $i$-th user's vector of a priori channel transfer factor estimates for the $n$-th OFDM symbol period during the $(n-1)$-th OFDM symbol period, which is denoted by $\hat{\mathbf{H}}_{a p r}^{(i)}[n]$, the vector of a posteriori channel transfer factor estimates $\tilde{\mathbf{H}}_{a p t}^{(i)}[n-1]$ is subjected to a unitary linear inverse transform $\tilde{\mathbf{U}}^{[f](i) H} \in \mathbb{C}^{K \times K}$, yielding the vector $\tilde{\mathbf{h}}_{\text {apt }}^{(i)}[n-1] \in \mathbb{C}^{K \times 1}$ of CIR-related a posteriori tap values:

$$
\tilde{\mathbf{h}}_{a p t}^{(i)}[n-1]=\tilde{\mathbf{U}}^{[f](i) H} \tilde{\mathbf{H}}_{a p t}^{(i)}[n-1] .
$$

From a statistical point of view the optimum unitary transform to be employed is the Karhunen-Loeve Transform (KLT) [1,2] with respect to the Hermitian spaced-frequency correlation matrix of a posteriori channel transfer factor estimates, which is given by $\mathbf{R}_{a p t}^{[f](i)}=E\left\{\tilde{\mathbf{H}}_{a p t}^{(i)} \tilde{\mathbf{H}}_{a p t}^{(i) H}\right\}$, when assuming the wide-sense stationarity of $\tilde{\mathbf{H}}_{a p t}^{(i)}[n]$. However, for practical reasons we assume here the employment of an arbitrary unitary matrix $\tilde{\mathbf{U}}^{[f](i)}$, which could be the DFT matrix W or one of the 'robust' transforms proposed by $\mathrm{Li}$ [3].

- In a second step linear $N_{t a p}^{[t]}$-tap filtering is performed in the time-direction separately for those $K_{0}$ number of CIR-related components of $\tilde{\mathbf{h}}_{a p t}^{(i)}$, for which the variance is significant. This is achieved by capitalizing on the current vector $\tilde{\mathbf{h}}_{a p t}^{(i)}[n-1]$ and the vectors $\tilde{\mathbf{h}}_{a p t}^{(i)}[n-\dot{n}], \dot{n}=2, \ldots, N_{t a p}^{[t]}$ of the previous $\left(N_{t a p}^{[t]}-1\right)$ number of OFDM symbols. As a result, in the case of estimation filtering [1] an improved estimate $\hat{\mathbf{h}}_{\text {apt }}^{(i)}[n-1]$ of $\mathbf{h}^{(i)}[n-1]$ is obtained, although this technique was not employed here. By contrast, in case of the prediction filtering employed here, an $a$ priori estimate $\hat{\mathbf{h}}_{a p r}^{(i)}[n] \in \mathbb{C}^{K \times 1}$ of $\mathbf{h}^{(i)}[n]$ is obtained. In mathematical terms this can be formulated as:

$$
\begin{aligned}
\hat{\mathbf{h}}_{a p r}^{(i)}[n] & =\sum_{l=0}^{K_{0}-1} \mathbf{I}_{1}^{(i)}[l] \sum_{n=1}^{N_{t a p}^{[t]}} \tilde{c}_{p r e}^{(i)}[n-1, l] \tilde{\mathbf{h}}_{a p t}^{(i)}[n-n](6) \\
& =\sum_{l=0}^{K_{0}-1} \mathbf{I}_{1}^{(i)}[l]\left\langle\tilde{\mathbf{h}}_{a p t}^{(i)}[n-1]\right\rangle \tilde{\mathbf{c}}_{\text {pre }}^{(i) *}[l]
\end{aligned}
$$

where the $l$-th sparse unity matrix $\mathbf{I}_{1}^{(i)}[l] \in \mathbb{C}^{K \times K}, l \in$ $\left\{0, \ldots, K_{0}-1\right\}$, exhibits a single unity entry only at the specific diagonal position, for which the variance of the associated CIR-related tap in the frame of $K$ CIR-related taps is significant. Furthermore, in Equation 6 the variable $\tilde{c}_{p r e}^{(i)}[\dot{n}-1, l] \in \mathbb{C}$ denotes the $(\dot{n}-1)$-th CIR-related tap prediction filter coefficient associated with the $l$-th significant CIR-related tap. In the context of the more compact notation of Equation 7, the matrix $\left\langle\tilde{\mathbf{h}}_{a p t}^{(i)}[n-\right.$ $1]\rangle \in \mathbb{C}^{K \times N_{t a p}^{[t]}}$ is defined as $\left\langle\tilde{\mathbf{h}}_{a p t}^{(i)}[n-1]\right\rangle=\left(\tilde{\mathbf{h}}_{a p t}^{(i)}[n-\right.$ $\left.1], \ldots, \tilde{\mathbf{h}}_{a p t}^{(i)}\left[n-N_{t a p}^{[t]}\right]\right)$, while the vector $\tilde{\mathbf{c}}_{\text {pre }}^{(i)}[k] \in \mathbb{C}^{N_{t a p}^{[t]} \times 1}$ is defined as $\tilde{\mathbf{c}}_{\text {pre }}^{(i)}[l]=\left(\tilde{c}_{\text {pre }}^{(i) *}[0, l], \ldots, \tilde{c}_{\text {pre }}^{(i) *}\left[N_{\text {tap }}^{[t]}-1, l\right]\right)^{T}$.

- In a last step the vector of CIR-related a priori tap estimates $\hat{\mathbf{h}}_{a p r}^{(i)}[n]$ is transformed back to the OFDM frequency-domain with the aid of the unitary matrix $\tilde{\mathbf{U}}^{[f](i)}$, yielding the vector of a priori channel transfer factor estimates $\hat{\mathbf{H}}_{a p r}^{(i)}[n]$ for the $n$-th OFDM symbol period:

$$
\hat{\mathbf{H}}_{a p r}^{(i)}[n]=\tilde{\mathbf{U}}^{[f](i)} \hat{\mathbf{h}}_{a p r}^{(i)}[n] .
$$

This vector of a priori channel transfer factor estimates is in turn employed in the detection stage during the $n$-th OFDM symbol period. Upon substituting Equation 5 into Equation 7 and by substituting the result into Equation 8 we obtain the following relation between the vector of a priori channel transfer factor estimates derived for the $n$-th OFDM symbol and the vectors of $a$ posteriori channel transfer factor estimates of the past $N_{t a p}^{[t]}$ number of OFDM symbols:

$$
\hat{\mathbf{H}}_{a p r}^{(i)}[n]=\sum_{l=0}^{K_{0}-1} \mathbf{T}_{1}^{(i)}[l]\left\langle\tilde{\mathbf{H}}_{a p t}^{(i)}[n-1]\right\rangle \tilde{\mathbf{c}}_{p r e}^{(i) *}[l],
$$

where $\mathbf{T}_{1}^{(i)} \in \mathbb{C}^{K \times K}$ is given by:

$$
\mathbf{T}_{1}^{(i)}[k]=\tilde{\mathbf{U}}^{[f](i)} \mathbf{I}_{1}^{(i)}[k] \tilde{\mathbf{U}}^{[f](i) H} .
$$


Note that $\left\langle\tilde{\mathbf{H}}_{a p t}^{(i)}[n-1]\right\rangle \in \mathbb{C}^{K \times N_{t a p}^{[t]}}$ is defined as $\left\langle\tilde{\mathbf{H}}_{a p t}^{(i)}[n-1]\right\rangle=$ $\left(\tilde{\mathbf{H}}_{a p t}^{(i)}[n-1], \ldots, \tilde{\mathbf{H}}_{a p t}^{(i)}\left[n-N_{t a p}^{[t]}\right]\right)$.

After having described the process of generating the vectors of $a$ posteriori and a priori channel transfer factor estimates in Sections IIIA and III-B, we will embark in Section IV on the task of optimizing the vectors $\tilde{\mathbf{c}}_{\text {pre }}^{(i)}[l], l=0, \ldots, K_{0}-1$ of predictor coefficients.

\section{RLS-BASED AdAPTATION OF THE CIR-RELATED TAP}

\section{PREDICTOR COEFFICIENTS}

In [4] it was demonstrated that the vectors $\tilde{\mathbf{c}}_{\text {pre }}^{(i)}[l], l=0, \ldots, K_{0}-1$ of predictor coefficients can be optimized off-line based on the concepts of 'robustness' as proposed by Li et al. [1] for the single-user scenario, upon taking into account the recursive structure of the estimator. However, in order to further improve the estimator's performance, a viable approach is adapt the predictor coefficients on a CIRrelated tap-by-tap basis, using the recursive least-squares (RLS) algorithm. The application of the RLS algorithm to the specific prediction task encountered will be described in the following ${ }^{1}$.

Recall that the $l$-th CIR-related tap's vector $\left.\tilde{\mathbf{c}}_{\text {pre }}[n, l]\right|_{\text {opt }} \in$ $\mathbb{C}^{N_{t a p}^{[t]} \times 1}$ of optimum predictor coefficients is determined by the Wiener equation [5], namely by:

$$
\left.\tilde{\mathbf{c}}_{p r e}[n, l]\right|_{o p t}=\tilde{\mathbf{R}}_{a p t}^{[t]-1}[n, l] \tilde{\mathbf{r}}_{a p t}^{[t]}[n, l],
$$

where $\tilde{\mathbf{R}}_{a p t}^{[t]}[n, l] \in \mathbb{C}^{N_{t a p}^{[t]} \times N_{t a p}^{[t]}}$ is the $l$-th CIR-related tap's estimated auto-correlation matrix and $\tilde{\mathbf{r}}_{a p t}^{[t]}[n, l] \in \mathbb{C}^{N_{t a p}^{[t]} \times 1}$ is the estimated cross-correlation vector, both of which are valid for the $n$-th OFDM symbol period. The estimate $\tilde{\mathbf{R}}_{a p t}^{[t]}[n, l]$ for the $n$-th OFDM symbol period could be obtained on the basis of the estimate $\tilde{\mathbf{R}}_{a p t}^{[t]}[n-1, l]$ associated with the $(n-1)$-th OFDM symbol period by evaluating [5]:

$\tilde{\mathbf{R}}_{a p t}^{[t]}[n, l]=\alpha_{\mathrm{RLS}} \tilde{\mathbf{R}}_{a p t}^{[t]}[n-1, l]+\left(1-\alpha_{\mathrm{RLS}}\right) \tilde{\mathbf{h}}_{a p t}[n-1, l] \tilde{\mathbf{h}}_{a p t}^{H}[n-1, l]$,

where $\tilde{\mathbf{h}}_{a p t}[n-1, l] \in \mathbb{C}^{N_{t a p}^{[t]} \times 1}$ is defined as the vector of $N_{t a p}^{[t]}$ number of past $l$-th CIR-related tap estimates starting with the OFDM symbol index $(n-1)$. Furthermore, in Equation 12 the variable $\alpha_{\mathrm{RLS}} \in$ $\mathbb{R}$ denotes the so-called forgetting factor [5]. Similarly, the estimate $\tilde{\mathbf{r}}_{a p t}^{[t]}[n, l]$ for the $n$-th OFDM symbol period can be obtained following the philosophy of Equation 12, yielding [5]:

$\tilde{\mathbf{r}}_{a p t}^{[t]}[n, l]=\alpha_{\mathrm{RLS}} \tilde{\mathbf{r}}^{[t]}[n-1, l]+\left(1-\alpha_{\mathrm{RLS}}\right) \tilde{h}_{a p t}^{*}[n, l] \tilde{\mathbf{h}}_{a p t}[n-1, l]$.

Instead of explicitly inverting the estimated auto-correlation matrix $\tilde{\mathbf{R}}_{a p t}^{[t]}[n]$ associated with the $n$-th OFDM symbol period, an iterative update strategy based on the matrix inversion lemma - also known as the Sherman-Morrison formula - or Woodbury's identity [5] can be invoked, which is known from the literature as the RLS algorithm [5]. In the context of our specific CIR-related tap prediction problem the RLS-algorithm is summarized below. Specifically, the so-called Kalman gain vector $\mathbf{k}[n, l] \in \mathbb{C}^{N_{t a p}^{[t]} \times 1}$ for the $n$-th OFDM symbol period is given by [5]:

$\mathbf{k}[n, l]=\frac{\left(1-\alpha_{\mathrm{RLS}}\right) \tilde{\mathbf{R}}_{a p t}^{[t]-1}[n-1, l] \tilde{\mathbf{h}}_{a p t}[n-1, l]}{\alpha_{\mathrm{RLS}}+\left(1-\alpha_{\mathrm{RLS}}\right) \tilde{\mathbf{h}}_{a p t}^{H}[n-1, l] \tilde{\mathbf{R}}_{a p t}^{[t]-1}[n-1] \tilde{\mathbf{h}}_{a p t}[n-1, l]}$

\footnotetext{
${ }^{1}$ For reasons of space enconomy we have omitted here the index ()$_{p}^{[i]}$ associated with the $i$-th user and the $p$-th receiver antenna element.
}

which is then employed in the process of updating the inverse of the CIR-related taps' auto-correlation matrix, namely [5]:

$$
\begin{aligned}
\tilde{\mathbf{R}}_{a p t}^{[t]-1}[n, l]= & \frac{1}{\alpha_{\mathrm{RLS}}}\left[\tilde{\mathbf{R}}_{a p t}^{[t]-1}[n-1, l]-\right. \\
& \left.-\mathbf{k}[n, l] \tilde{\mathbf{h}}_{a p t}^{H}[n-1, l] \tilde{\mathbf{R}}_{a p t}^{[t]-1}[n-1, l]\right]
\end{aligned}
$$

Furthermore, the CIR-related tap predictor coefficient vector for the $n$-th OFDM symbol period is given by [5]:

$$
\begin{aligned}
\left.\tilde{\mathbf{c}}_{p r e}[n, l]\right|_{o p t}= & \left.\tilde{\mathbf{c}}_{p r e}[n-1, l]\right|_{o p t}+\mathbf{k}[n, l]\left[\tilde{h}_{a p t}[n, l]-\right. \\
& \left.-\tilde{\mathbf{c}}_{p r e}^{H}[n-1, l] \tilde{\mathbf{h}}_{a p t}[n-1, l]\right]^{*},
\end{aligned}
$$

where the term in brackets denotes the prediction error associated with the $n$-th OFDM symbol period. A standard approach for initializing the RLS algorithm [5] is that of assuming an inverse correlation matrix having a diagonal shape defined as:

$$
\tilde{\mathbf{R}}_{a p t}^{[t]-1}[0, l]=\frac{1}{\varepsilon_{\mathrm{RLS}, 0}} \mathbf{I}
$$

where the specific choice of $\varepsilon_{\mathrm{RLS}, 0} \in \mathbb{R}$ is less critical in our application, than the specific value of the forgetting-factor $\alpha_{\mathrm{RLS}}$. A plausible choice for $\tilde{\mathbf{c}}_{\text {pre }}[0, l]$ is for example $(1,0, \ldots, 0)^{T}$, which corresponds to the case of zero-forcing based one-tap prediction.

\section{Performance Assessment}

\section{A. RLS-Adaptive DDCE for Single-User OFDM}

In order to demonstrate the applicability of the RLS algorithm to the problem of CIR-related tap prediction in the context of DDCE we will first focus on the single-user scenario. In Figures 3 and 4 we have portrayed the evolution of the a priori channel estimation MSE versus the OFDM symbol index for an arbitrary time segment commencing with an initial vector of prediction coefficients given by $\tilde{\mathbf{c}}_{\text {pre }}^{(i)}[0, l]=$ $(1,0, \ldots, 0)^{T}$, where $l=0, \ldots, K_{0}-1$. Here we have employed the sample-spaced indoor WATM channel model of [6], where the highest CIR tap delay is given by $11 T_{s}$. Hence, the number of significant CIRrelated taps was chosen as $K_{0}=12$. Furthermore, the CIR-related tap predictor's range was equal to $N_{t a p}^{[t]}=4$. Note that for different time segments the specific MSE evolution is potentially different from that of Figures 3 and 4, but obey the same general trend. Here we have investigated the influence of the Kalman forgetting factor $\alpha_{\mathrm{RLS}}$ and of the OFDM symbol normalized Doppler frequency on the a priori channel estimation MSE performance. Specifically, in Figure 3 the OFDM symbol normalized Doppler frequency was set to $F_{D}=0.007$, while the forgetting factor $\alpha_{\text {RLS }}$ was varied. We observe in Figure 3 that for lower values of $\alpha_{\text {RLS }}$ a faster adaptation is achieved, while the residual error after adaptation is potentially higher, than that achieved with the aid of a forgetting factor of a higher value, although the latter effect is not explicitly visible in Figure 3 due to the limited time span. By contrast, in Figure 4 we have plotted the a priori channel estimation MSE for various OFDM symbol normalized Doppler frequencies, $F_{D}$, while keeping the forgetting factor $\alpha_{\text {RLS }}$ constant. As expected, the $a$ priori estimation MSE is increased in scenarios having a higher OFDM symbol normalized Doppler frequency, while the speed of adaptation was almost identical for the different scenarios. Note that for values of $\alpha_{\text {RLS }}$ that are significantly lower than those employed in Figure 4, the RLS predictor may potentially become unstable. Specifically, in our experiments a value of $\alpha_{\mathrm{RLS}}=0.77$ was just acceptable, and yielded the highest speed of convergence while at the same time potentially exhibiting the highest residual error after adaptation. 
Fr.-Inv. Fad. SWATM, 1 Rec.-Antenna, 1 User, MPSK

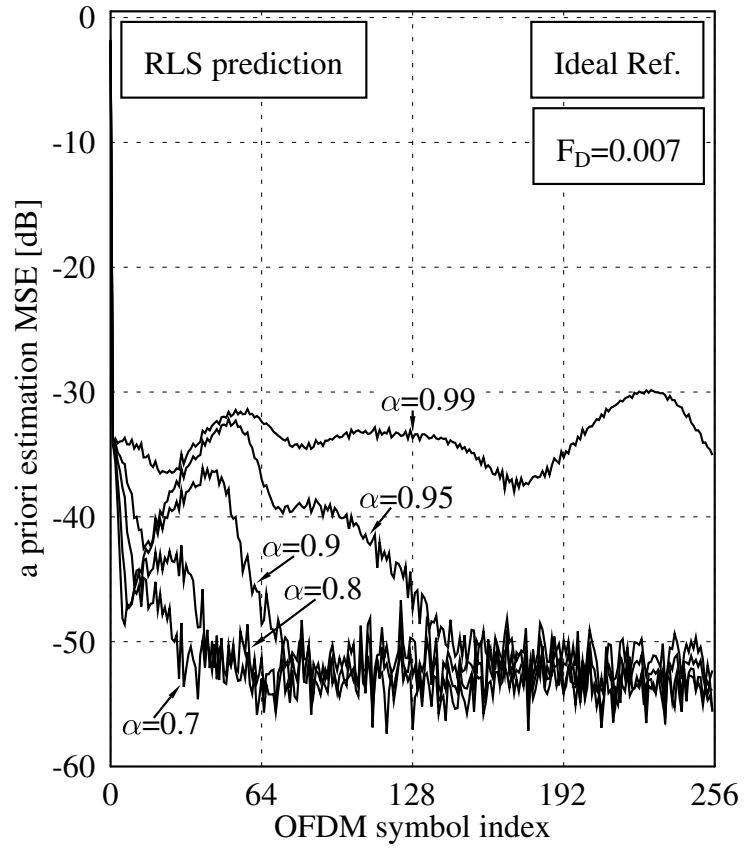

Fig. 3. Evolution of the a priori channel estimation MSE observed with the aid of the RLS prediction assisted DDCE in the single-reception antenna based single-user scenario for a specific time-segment associated with the samplespaced indoor WATM channel of [6], as a function of the OFDM symbol index, parameterized with the forgetting factor $\alpha=\alpha_{\mathrm{RLS}}$, for a fixed OFDM symbol normalized Doppler frequency of $F_{D}=0.007$; the RLS predictor's startup constant was $\varepsilon_{\mathrm{RLS}, 0}=0.1$, the $N_{\text {tap }}^{[t]}$ number of CIR predictor taps was equal to four and the SNR at the reception antennas was assumed to be $40 \mathrm{~dB}$; furthermore the number of significant CIR-related taps was $K_{0}=12$ and the number of subcarriers was $K=512$.

\section{B. RLS-Adaptive PIC-Assisted DDCE for Multi-User OFDM}

Let us now focus our attention on the standard multi-user OFDM scenario of four simultaneous users, each equipped with one transmit antenna, while at the basestation (BS) a four-element antenna array is employed. The channel between each transmit-receive antenna pair characterized in terms of its sample-spaced impulse response - and the OFDM parameters are fixed to those used by the indoor WATM system of [6]. Two detection techniques are invoked in our study, namely, the MMSE [7-9] and the M-SIC (M=2) [8-10] detection techniques. The corresponding BER- and a priori channel estimation MSE simulation results - following the initial adaptation ${ }^{6}$ of the predictor coefficients are portrayed in Figures 5 and 6, respectively.

In the context of the BER performance assessment shown in Figure 5 we observe that with the aid of the imperfect channel estimates produced by the RLS-adaptive PIC-assisted DDCE almost the same performance is achieved, as in case of employing ideal channel knowledge. This is particularly true for the powerful M-SIC detection algorithm, which produces relatively reliable symbol decisions and hence also a reliable remodulated reference for the RLS-adaptive PICassisted DDCE. By contrast, a slight BER degradation is observed, when using the less powerful MMSE detection scheme, particularly for the range of SNRs up to $5 \mathrm{~dB}$. For higher SNRs the BER performance is also almost identical to that when using perfect channel estimates.

The benefits of a more reliable remodulated reference used in the RLS-adaptive PIC-assisted DDCE become even more evident from the

\footnotetext{
${ }^{6}$ The initial adaptation of the predictor coefficients was observed in Figures 3 and 4. After the adaptation the a priori channel estimation MSE fluctuates around its specific mean value.
}

Fr.-Inv. Fad. SWATM, 1 Rec.-Antenna, 1 User, MPSK

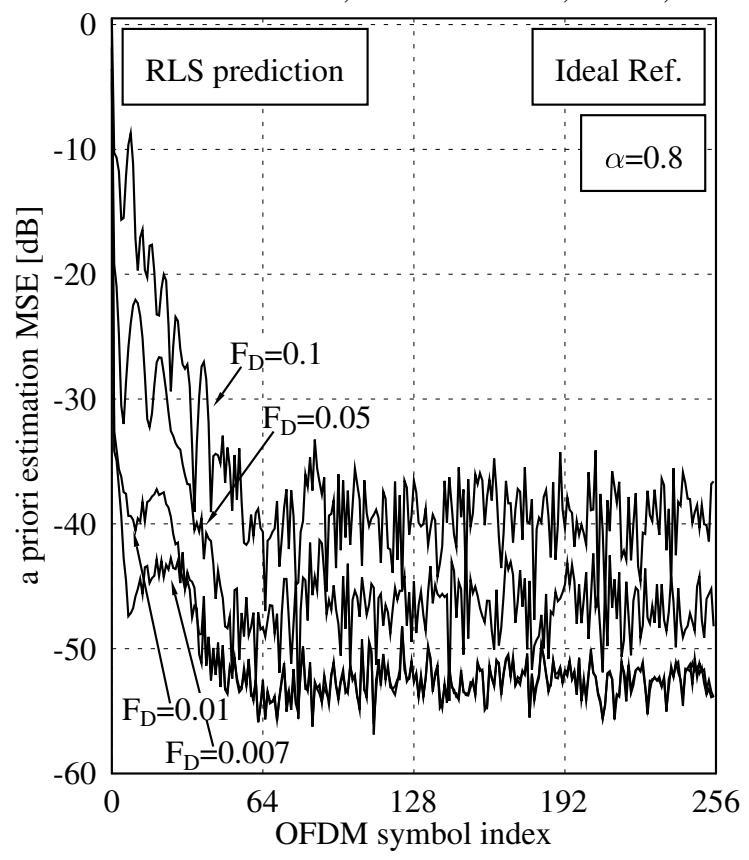

Fig. 4. Evolution of the a priori channel estimation MSE observed with the aid of the RLS prediction assisted DDCE in the single-reception antenna based single-user scenario for a specific time-segment associated with the samplespaced indoor WATM channel of [6], as a function of the OFDM symbol index; parameterized with the OFDM symbol normalized Doppler frequency $F_{D}$ and for a fixed forgetting factor of $\alpha_{\mathrm{RLS}}=0.8$; in both cases the RLS predictor's startup constant was $\varepsilon_{\mathrm{RLS}, 0}=0.1$, the $N_{\text {tap }}^{[t]}$ number of CIR predictor taps was equal to four and the SNR at the reception antennas was assumed to be $40 \mathrm{~dB}$; furthermore the number of significant CIR-related taps was $K_{0}=12$ and the number of subcarriers was $K=512$.

MSE performance results shown in Figure 6. Here we observe a significant MSE reduction, when employing the M-SIC assisted generation of the remodulated reference, rather than that of the MMSE detector. In our specific example, which employs the sample-spaced three-path indoor WATM CIR, all of the channel's energy is concentrated in three CIR-related taps, namely those at zero, six and eleven sampling period delays. BY contrast, at all other tap positions within the CIR window of the first $K_{0}=12$ taps, the RLS-based adaptive predictor succeeds in effectively reducing the noise without setting these taps by 'brute force' to zero. Hence the maximum noise reduction factor is about $3 / 512$. Note however again that in the more realistic scenario of a non-sample-spaced CIR in conjunction with employing the unitary DFT matrix $\mathbf{W}$ for transforming the least-squares channel transfer factor estimates from the frequency-domain to the CIR-related domain, the noise reduction is more moderate due to the effects of CIR-related domain leakage as it was discussed by van de Beek et al. [11]. More explicitly, the energy conveyed by the channel is rather spread across the different CIR-related taps.

Finally, let us comment on the specific choice of the forgetting factor of $\alpha_{\mathrm{RLS}}=0.95$. As suggested during our investigations of RLS based adaptive prediction-assisted DDCE employed in single-user OFDM systems in Section V, for small values of $\alpha_{\mathrm{RLS}}$, the predictor may become unstable, as a result of which an excessive estimation MSE is observed. Our experiments conducted in the context of the PIC-assisted DDCE of Figure 2 further underlined that the appropriate range of $\alpha_{\text {RLS }}$ values has to be re-optimized when invoking an imperfect, potentially error-contaminated reference. Again, relatively small values of $\alpha_{\text {RLS }}$ yield a fast convergence, but also a high sensitivity to erroneous symbol decisions, while for higher values of $\alpha_{\text {RLS }}$ the opposite is true. 


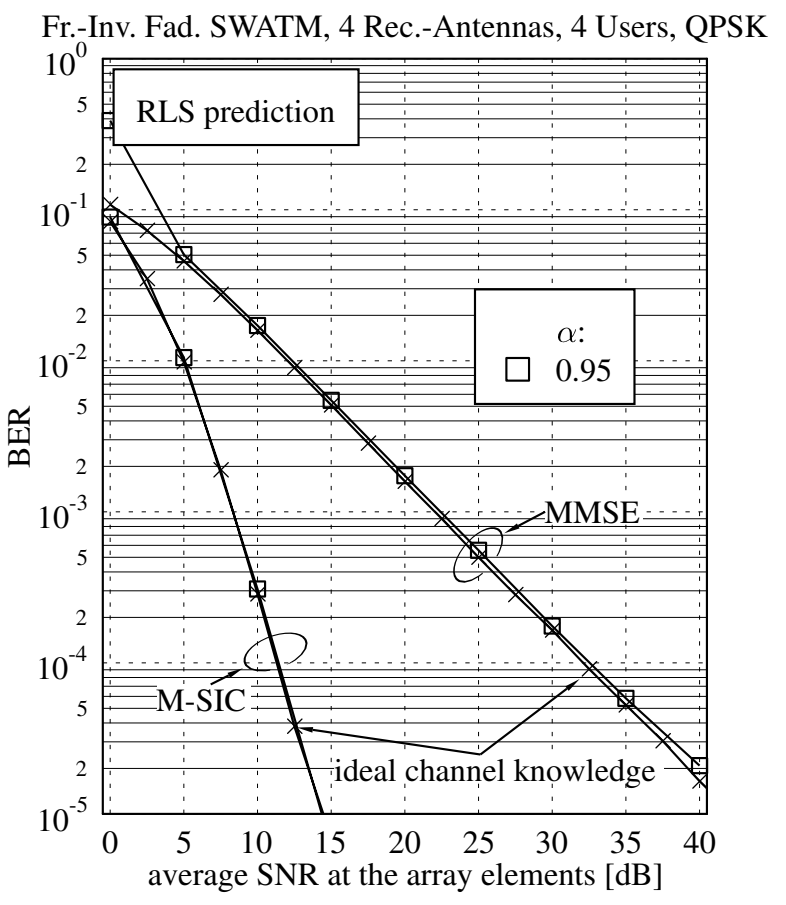

Fig. 5. BER versus SNR performance associated with the CIR-related tap-by-tap based RLS-adaptive PIC-assisted DDCE of Figure $2^{3}$ in the context of a scenario of four receiver antennas at the BS and four simultaneous users, each equipped with one transmit antenna; the channel between each transmitter-receiver antenna pair is characterized in terms of its sample-spaced impulse response and OFDM parameters by the indoor WATM channel- and system parameters of [6]; the OFDM symbol normalized Doppler frequency was $F_{D}=0.007$; furthermore, the number of significant CIR-related taps was $K_{0}=12$ and the number of subcarriers was $K=512$; MMSE- as well as M-SIC ( $M=2)$ detection was employed at the receiver and both an ideal, errorfree reference and an imperfect, error-contaminated reference was invoked in the DDCE; the RLS-specific forgetting factor was set to $\alpha=\alpha_{\mathrm{RLS}}=0.95$.

A choice of $\alpha_{\mathrm{RLS}}=0.95$ was deemed reasonable in our application.

\section{Conclusions}

In this paper the application of the RLS algorithm to adapting the CIR-related tap predictors' coefficients in the context of PIC-assisted DDCE designed for OFDM systems employing multiple transmit antennas was studied. We characterised the influence of the RLS-specific forgetting factor $\alpha_{\mathrm{RLS}}$ and that of the channel's OFDM symbol normalized Doppler frequency $F_{D}$ on the estimator's convergence properties in the single-user scenario, as shown in Figures 3 and 4. Furthermore, the estimator's convergence experienced in the context of a multi-user scenario of four transmit antennas and four BS receiver antennas was confirmed in Figures 5 and 6 . In the context of the sample-spaced three-path CIR associated with the indoor WATM channel model of [6], the BER performance achieved using the proposed estimator was almost indistinguishable from that when capitalizing on ideal channel knowledge. In the context of a non-sample spaced CIR a similarly good performance is expected upon invoking the 'robust' transforms proposed by Li [3]. This is in contrast to the performance expected when using the DFT matrix $\mathbf{W}$, which is responsible for the effects of CIR-related domain leakage [11].

\section{REFERENCES}

[1] Y. Li, L. J. Cimini, and N. R. Sollenberger, "Robust Channel Estimation for OFDM Systems with Rapid Dispersive Fading Channels," IEEE Transactions on Communications, vol. 46, pp. 902-915, April 1998.
Fr.-Inv. Fad. SWATM, 4 Rec.-Antennas, 4 Users, QPSK

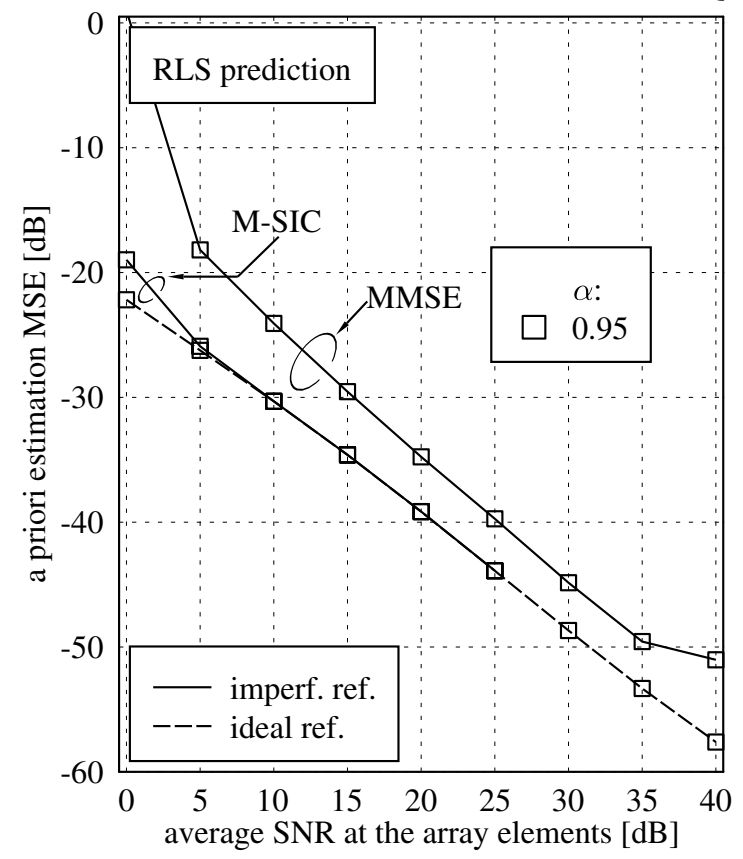

Fig. 6. A priori channel estimation MSE versus SNR performance associated with the CIR-related tap-by-tap based RLS-adaptive PIC-assisted DDCE of Figure $2^{5}$ in the context of a scenario of four receiver antennas at the BS and four simultaneous users, each equipped with one transmit antenna; the channel between each transmitter-receiver antenna pair is characterized in terms of its sample-spaced impulse response and OFDM parameters by the indoor WATM channel- and system parameters of [6]; the OFDM symbol normalized Doppler frequency was $F_{D}=0.007$; furthermore, the number of significant CIR-related taps was $K_{0}=12$ and the number of subcarriers was $K=512$; MMSE- as well as M-SIC (M=2) detection was employed at the receiver and both an ideal, error-free reference and an imperfect, error-contaminated reference was invoked in the DDCE; the RLS-specific forgetting factor was set to $\alpha=\alpha_{\mathrm{RLS}}=0.95$.

[2] O. Edfors, M. Sandell, J.-J. v. d. Beek, S. K. Wilson, and P. O. Börjesson, "OFDM Channel Estimation by Singular Value Decomposition," IEEE Transactions on Communications, vol. 46, pp. 931-939, April 1998.

[3] Y. Li and N. R. Sollenberger, "Clustered OFDM with Channel Estimation for High Rate Wireless Data," IEEE Transactions on Communications, vol. 49, pp. 2071-2076, December 2001.

[4] M. Münster and L. Hanzo, "Parallel Interference Cancellation Assisted Decision-Directed Channel Estimation for OFDM Systems Using Multiple TRansmit Antennas," IEEE Transactions on Wireless Communications, submitted, 2002.

[5] T. K. Moon and W. C. Stirling, Mathematical Methods and Algorithms for Signal Processing. Prentice Hall, 2000.

[6] L. Hanzo, W. Webb, and T. Keller, Single-and Multi-carrier Quadrature Amplitude Modulation. IEEE Press- John Wiley, April 2000.

[7] Y. Li and N. R. Sollenberger, "Adaptive Antenna Arrays for OFDM Systems with Cochannel Interference," IEEE Transactions on Communications, vol. 47, pp. 217-229, February 1999.

[8] M. Münster and L. Hanzo, "Co-Channel Interference Cancellation Techniques for Antenna Array Assisted Multiuser OFDM Systems," in Proceedings of 3G'2000 Conference, vol. 1, (London, Great Britain), pp. 256-260, IEE, March 27-29 2000.

[9] P. Vandenameele, L. V. D. Perre, M. G. E. Engels, B. Gyselinckx, and H. J. D. Man, "A Combined OFDM/SDMA Approach," IEEE Journal on Selected Areas in Communications, vol. 18, pp. 2312-2321, Nov. 2000.

[10] G. D. Golden, G. J. Foschini, R. A. Valenzuela, and P. W. Wolniansky, "Detection Algorithms and Initial Laboratory Results using VBLAST Space-Time Communication Architecture," IEE Electronics Letters, vol. 35, pp. 14-16, January 1999.

[11] J.-J. van de Beek, O. Edfors, M. Sandell, S. K. Wilson, and P. O. Börjesson, "On channel estimation in OFDM systems," in Proceedings of Vehicular Technology Conference, vol. 2, (Chicago, IL USA), pp. 815819, IEEE, July 1995. 